\title{
EMDR intervention after a disaster: The Morandi Bridge collapse
}

\author{
Isabel Fernandez, ${ }^{1}$ Chiara Callerame ${ }^{2}$ \\ ${ }^{1}$ Center for Studies and Research in Psychotraumatology; ${ }^{2}$ EMDR Center for Eating Disorders, Milan, Italy
}

\begin{abstract}
Survivors involved in natural or man-made disasters can develop a range of psychological problems including Post-Traumatic Stress Disorder (PTSD), depression, other forms of psychological distress, and a poor quality of life. Therefore, the principal focus of early psychological interventions, according to World Health Organization, is to provide a rapid and effective therapeutical approach like EMDR therapy. The EMDR approach enables the assimilation and integration of the various aspects of a traumatic experience at a somatic, sensorial, cognitive, behavioral and emotional level. In this article will be presented data of 47 patients collected after the partial collapse of Morandi bridge, in Genoa, in August 2018. Survivors were treated applying the Recent Traumatic Episode Protocol (R-TEP); in order to evaluate the effect of the traumatic event and to monitor the intervention outcome, the Impact of Event Scale- Revised (IES-R) was administered to each person pre and after EMDR treatment. Results show that regardless the number of sessions and the time elapsed after the disaster in which they were performed, EMDR significantly reduced participants' IES-R scores from pre-treatment to posttreatment. Clinical implications and limits of the study will be discussed.
\end{abstract}

\section{Introduction}

Life events, such as natural or man-made disasters, are considered extreme and traumatic experiences, which have a strong impact on the survivors' quality of life. The DSM 5 considers these

Correspondence: Chiara Callerame, Faculty of Communication Sciences, Institute for Public Com-munication, University of Lugano; and EMDR Center for Eating Disorders (Centro EMDR per l'Anoressia), via Pergolesi 14, 10124, Milan, Italy.

E-mail: chiara.callerame@gmail.com

Key words: EMDR; disaster; PTSD.

Conflict of interests: the authors declare no conflict of interests.

Received for publication: 11 October 2019.

Revision received: 29 November 2019.

Accepted for publication: 2 December 2019

This work is licensed under a Creative Commons Attribution 4.0 License (by-nc 4.0)

(C) Copyright: the Author(s), 2019

Licensee PAGEPress, Italy

Emergency Care Journal 2019; 15:8634

doi:10.4081/ecj.2019.8634 experiences as major trauma and defines them as experiences that threaten one's physical safety and integrity (or that of a loved and close relative or friend). ${ }^{1}$ Survivors involved in such experiences can develop a range of psychological problems including Acute Stress Disorder ${ }^{1}$ in the immediate aftermath of the traumatic event, Post-Traumatic Stress Disorder (PTSD), depression, ${ }^{2-6}$ other forms of psychological distress, ${ }^{7,8}$ and poorer quality of life. ${ }^{9,10}$

Epidemiological research suggests that $60-80 \%$ of individuals with Acute Stress Disorder (ASD) develop PTSD and a third of subjects with PTSD symptoms remain symptomatic for six years or longer. ${ }^{11,12}$ Furthermore, in over $80 \%$ of patients' PTSD is comorbid with at least one other mental disorder, ${ }^{12,13}$ major depressive disorder being one of the most common one. ${ }^{14}$

The majority of people who develop PTSD do not initially manifest symptomatology, ${ }^{15}$ hence one of the greatest challenges is to predict the possible clinical worsening occurring over time. According to Jarero and Uribe, ${ }^{16,17}$ a lack of safety during the aftermath, along with trauma reminders, generally interferes with the reorganizing consolidation process of a traumatic memory. ${ }^{18}$ Thus, the memory network remains in a permanent excitatory state, sensitizing and expanding with each subsequent stressful event. Over time, unprocessed and unresolved trauma-related psychological effects along with the cumulative effect due to other forms of intervening stress and reminders of the original trauma facilitate the development of PTSD, depression and substance abuse. ${ }^{19}$

Perceptions, bodily sensations, emotions and expectations related to the memories of traumatic events tend to be crystallized in memory networks which remain non-processed in the brain. The remaining memories not coded into neural networks are associated to mal-adaptive sensations, beliefs and images. Andreski et al. reported that, of all the psychiatric disorders, PTSD has an overwhelming impact on almost every aspect of the life of trauma survivors and is strongly correlated to somatization and medically unexplained pain..$^{20,21}$

Given these considerations it is evident the importance of a prompt and adequate intervention in the aftermath of a stressful event. Many trauma survivors, in fact, will benefit of the help from their communities, families and other forms of support to prevent $\mathrm{PTSD}^{1}$ or long-term psychological morbidity. ${ }^{22}$

The main focus of early psychological interventions after a traumatizing event for individuals, families and groups, according to WHO are: i) supplying consolation and concern, in order to reduce hypervigilance states (create a safe environment) and ii) provide secure information to all those involved ${ }^{23}$ adopting a rapid and effective therapeutical approach like EMDR therapy. The standard EMDR protocol consists of eight phases, ${ }^{24}$ which enable the assimilation and integration of the various aspects of a traumatic experience at a somatic, sensorial, cognitive, behavioral and emotional level. EMDR is a psychotherapeutic frame which activates the brain's natural information processing ability, based on the adaptive integration of dysfunctionally stored emotional experience. ${ }^{25-29}$ It is a short-lasting trauma-focused psychotherapy and its core element is the bilateral sensorial stimulation aimed at favoring the reprocessing traumatic memories. The effectiveness of EMDR 
in treating PTSD was demonstrated in randomized controlled studies in which EMDR was more effective than no treatment at all, ${ }^{30-}$ ${ }^{32}$ and more effective compared to pharmacotherapy alone. ${ }^{33}$ These findings have been corroborated by the results of five meta-analy$\operatorname{ses}^{34-39}$ and EMDR has been found to be equally effective ${ }^{40}$ or rather superior to behavioral or cognitive-behavioral methods in terms of trauma resolution. ${ }^{41,42}$ In fact, while EMDR requires only 8 sessions and no homework assignment, CBT is much more complex and requires exposure to in vivo sessions as well as many hours of homework. Furthermore, it was shown that $100 \%$ of single trauma victims who underwent EMDR treatment did not manifest PTSD symptoms after receiving an average of 6 sessions, 50 min each. ${ }^{43}$ The Guidelines of the WHO state that although CBT and EMDR therapy are recommended for the treatment of PTSD in children, adolescents and adults, EMDR therapy aims at reducing subjective distress and empowers the adaptive cognition associated to the traumatic event without the need of a detailed description of it. The last edition of the International Society for Traumatic Stress Studies (ISTSS), in October 2018 describes EMDR as having Emerging evidence for adults as early intervention, establishing that a single session of EMDR within the first 3 months of a traumatic event can prevent PTSD. EMDR received also from these guidelines a Standard recommendation within the first 3 months in multiple sessions, with the statement that is equal to CBT-Trauma Focused and to Cognitive therapy. No interventions are considered strong by these guidelines for the acute phase, but all therapies are considered standard.

Various EMDR protocols have been proposed by different authors. ${ }^{44-64}$ In 2008, Shapiro and Laub developed the Recent Traumatic Episode Protocol (R-TEP). ${ }^{47,59,60}$ In a randomized controlled trial, 17 survivors with posttraumatic distress were treated reporting a significant decrease of symptoms, which was maintained at 3 months follow-up. ${ }^{65}$ Moreover, in a study of Saltini and colleagues, R-TEP was provided within three months of the traumatic event to a large sample of individuals exposed to the earthquake that hit Northern Italy in 2012 suggesting that EMDR is a useful treatment option in response to a disaster crisis and in reducing psychological distress of acutely traumatized individuals within the context of a natural disaster. ${ }^{66}$

Therefore, these data confirm previous studies in which EMDR was applied in different kinds of trauma, ${ }^{52-54}$ and emergency situations, underlining its effectiveness in the immediate aftermath. ${ }^{51,55-57}$

\section{EMDR in an emergency context in Italy: the Morandi bridge collapse}

On 14 August 2018, a partial collapse of Morandi bridge, in Genoa, involved about thirty vehicles and ten heavy vehicles killing 43 people. In that emergency context, the EMDR Italian Association was asked by the Town Hall of Genova and by the National Health Service to intervene immediately in the aftermath with a team of 48 psychotherapists specialized in the management of stress in the acute phase.

Interventions were directed to survivors, families of the victims, rescuers and to people who lived in the red zone and were evacuated losing their daily lives. A few days after the disaster, they started to manifest posttraumatic symptoms, feeling fearful and realizing the consequences of the crash. All this was predisposing to a long-term impact on mental and physical health, in particular for those under the age of 18, a population at very high risk and needing specific support.

In an emergency context it is important to consider as a priority the needs of the population, in order to meet their needs with spe- cific humanitarian intervention.

Despite the limitations due to the emergency context, prior to data collection, all subjects received complete information concerning the rationale and effectiveness of EMDR and the study procedures and gave written informed consent for their participation in the study.

This study was conducted in accordance with the Declaration of Helsinki (2001), under the approval of the research guidelines of the Italian EMDR Association and Article 10 of the National Board of Italian Psychologists Code of Ethics for the Psychologist. Moreover, regarding the ethical issues, the study was implemented following the request for intervention by the City of Genoa and upon the approval of the ethic panel of the EMDR Italian Association (Prot.EMDR_Genoa, 1.0).

Survivors were provided with a total number of EMDR sessions ranging from two to six in the immediate aftermath of the disaster applying the Recent Traumatic Episode Protocol (RTEP). ${ }^{47,59,60} \mathrm{~A}$ minimum of two sessions was deemed necessary as follow-up after the first session is considered essential. Survivors were treated on consecutive days. The treatment program included the preparation phase, the installing the safe place technique, and the R-TEP protocol in all steps. After processing the memory of the worst aspects of the disaster, the present and future template was completed.

EMDR therapists were able to assist pro bono around 150 people with a total number of sessions of around 1000 in 3 months (acute phase), continuing the interventions, when needed, until November. In order to evaluate the effect of traumatic event and to monitor intervention outcome, the Impact of Event Scale-Revised (IES-R) was administered to each person pre and after EMDR treatment, during the first and the last EMDR session. ${ }^{61}$

The IES-R assesses the subjective distress perceived in relation to a potentially traumatic event. Each item is assessed according to a scale from 0 to 4 points, where 0 represents absence of relevance to the item and 4 extreme relevance. ${ }^{61}$

\section{Results}

It was possible to perform complete and reliable analyses in 47 patients (18 males, 29 females; mean age 54 years, \pm 16.4 ), in which all parameters under consideration were correctly collected (Table 1).

The limited number of patients on which reliable analyses were based was mainly due to the difficulty to perform psychological tests in an emergency field and in a dramatic psychological condition, and also to the dropouts at follow-up for personal and logistic reasons. Regardless the number of sessions (mean 2.8 \pm 1.5 ) and the time elapsed after the disaster in which they were performed (14.3 \pm 8.4 days), EMDR significantly reduced participants' IES-R scores which decreased from $52.5 \pm 17$ pre-treatment to $22.9 \pm 19.6$ post-treatment $(\mathrm{p}<0.05)$.

Table 1. Means and standard deviations.

\begin{tabular}{lccc} 
& N & M & SD \\
AGE & 47 & 54 & 16.4 \\
TIME_ELAPSED & 47 & 14.3 & 8.4 \\
\hline NUMBER_SESSION & 47 & 2.8 & 1.5 \\
T1_IESR & 47 & 52.5 & 17 \\
\hline T2_IESR & 47 & 22.9 & 19.6 \\
\hline
\end{tabular}




\section{Discussion}

This study evaluated the effects of EMDR provided to a sample of individuals exposed to the collapse of Morandi Bridge in 2018. The analysis of the IES-R scale makes it possible to hypothesize the efficacy of the treatment in reducing the number of subjects with PTSD symptomatology, as seen in the comparisons between the first and final sessions of the treatment.

Even if on hypothetic basis due to the limits of the research, our results suggest that patients' response to treatment could depend on their characteristics, instead of the number and the time sequence of the sessions. Our results, therefore, make it possible to hypothesize that EMDR therapy could be considered as a flexible treatment in an emergency context: some patients could need two, three or even six sessions to resolve the symptoms and the treatment could be done in consecutive days or delayed during the month.

The results make it also possible to hypothesize the absence of a possible time dependent effect, between the collapse and the intervention. Indeed, the data collected show that all the participants recovered their normal functioning, regardless the time past since the event for which they were treated.

In conclusion, independently if participants were seeking for help and treated at the very beginning of the aftermath of the event or at the end of the acute phase, they responded significantly to the EMDR treatment, even after a very small number of sessions.

\section{Limits of this study}

Being an observational study, this work shows an association between early EMDR therapy and patients' benefit but cannot demonstrate a causal effect. The humanitarian nature of this research, in fact, determined several limits such as the limited sample number, the impossibility to implement a randomized condition and to have a control group.

We must also acknowledge that only 47 of 150 people could be completely evaluated; we have no reason to believe that the group of patients who did not complete all the psychological tests significantly differed in their characteristics from the ones that underwent a complete evaluation, but we don't have the certainty that other factors have been involved and, due to research limitations, they may not have been evaluated.

\section{Conclusions}

This study evaluated the effects of EMDR provided to a sample of individuals exposed to the collapse of Morandi Bridge in 2018; however, the lack of a randomized control group and the absence of follow up do not allow us to draw any conclusion about the statistical significance of the effectiveness of the R-TEP protocol. Furthermore, given the emergency context, assessment tools were administered by the same therapists who carried out the treatment, and diagnosis was defined on the basis of clinical judgement. However, it is important to keep in mind some specific features of the EMDR treatment, which make it particularly useful in emergency contexts. International guidelines, in fact, underline the importance of early and specialized intervention within emergency contexts and in the subsequent stages, to ensure reduction of distress and prevent future physical and psychopathological disorders in adults and children. ${ }^{62,63}$ Given its rapid efficacy in treating children and adults with ASD and PTSD symptomatology, EMDR has been chosen in many crisis managements as the right provider for mental health intervention. Furthermore, EMDR interventions can be very helpful in reducing secondary traumatic stress and emotional responses in institutional representatives and emergency workers, to safeguard their wellbeing and enhance their capacity to deliver help in the post-disaster intervention to their community. EMDR interventions can be implemented in the aftermath of largescale disasters after a traumatic event. Psychological teams of EMDR clinicians are highly trained to manage the emergency within the first hours, delivering immediate emotional support to survivors, including psychological first aid by focusing on strength, skill and resilience building.

EMDR limits the use of avoidance as a main coping strategy and stimulates more adaptive answers; it is an extremely flexible tool and does not require a rigid setting, nor homework assignments and can be administered by different therapists to the same client in consecutive days. Processing the recent traumatic experience of the disaster, addressing present triggers and future templates are the key elements of the EMDR method which can be dealt with in the ongoing aftermath.

This is consistent with the Action Plan for Mental Health of the World Health Organization (2012) and with its guidelines for the treatment of stress-related disorders (2013). Contingency planning should address a time span that extends from immediate, to middle and long-term interventions. EMDR interventions can be focused on individuals, families, organizational teams, or even the entire community.

Following its inclusion within the recommendation of the WHO guidelines and ISTSS guidelines and given the great body of EMDR early interventions implemented worldwide in the past two decades, it is evident that EMDR interventions should become part of both contingency and emergency plans for aftermath recovery in all its phases following a disaster.

As suggested by Shapiro, however, further studies and scientific evidence in emergency contexts are auspicable. ${ }^{67}$

\section{References}

1. American Psychiatric Association. Diagnostic and Statistical Manual of Mental Disorders (DSM 5). Washington DC: American Psychiatric Association; 2013.

2. Norris FH, Friedman MJ, Watson PJ, et al. 60,000 disaster victims speak: Part 1. An empirical review of the empirical literature, 1981-2001. Psychiatr 2002;65:207-39.

3. Wu Z, Xu J, He L. Psychological consequences and associated risk factors among adult survivors of the 2008 Wenchuan earthquake. BMC Psychiatr 2014;14:126.

4. Wu Z, Xu J, Sui Y. Posttraumatic stress disorder and posttraumatic growth coexistence and the risk factors in Wenchuan earthquake survivors. Psychiatric Research 2016;23:49-54.

5. Xu J, Song X. Posttraumatic stress disorder among survivors of the Wenchuan earthquake 1 year after: Prevalence and risk factors. Compr Psychiat 2011;52:431-7.

6. Zhang Z, Shi Z, Wang L, Liu M. One year later: Mental health problems among survivors in hard-hit areas of the Wenchuan earthquake. Public Health 2011;125:293-300.

7. Oyama M, Nakamura K, Suda Y, Someya T. Social network disruption as a major factor associated with psychological distress 3 years after the 2004 Niigata-Chuetsu earthquake in Japan. Environ Health Prev 2012;17:118-23.

8. Toyabe S, Shioiri T, Kuwabara H, et al. Impaired psychological recovery in the elderly after the Niigata-Chuetsu 
Earthquake in Japan: A population-based study. BMC Public Health 2006;6:230.

9. Tsai KY, Chou P, Chou FHC, et al. Threeyear follow-up of the relationship between posttraumatic stress symptoms and quality of life among earthquake survivors in Yu-Chi, Taiwan. J Psychiat Res 2007;41:90-6.

10. Wang XD, Gao L, Shinfuku N, et al. Longitudinal study of earthquake-related PTSD in a randomly selected community sample in North China. Am J Psychiat 2000;157:1260-6.

11. Andrews B, Brewin CR, Philpott R, Stewart L. Delayed-onset posttraumatic stress disorder: A systematic review of the evidence. Am J Psychiat 2007;164:1319-26.

12. Kessler R, Sonnega A, Bromet E, et al. Posttraumatic stress disorder in the national comorbidity survey. Arch Gen Psychiat 1995;52:1048-60.

13. Creamer M, Burgess P, McFarlane AC. Post-traumatic stress disorder: Findings from the Australian national survey of mental health and well-being. Psychol Med 2001;31:1237-47.

14. Rytwinski NK, Scur MD, Feeny NC, Youngstrom EA. The cooccurrence of major depressive disorder among individuals with posttraumatic stress disorder: A meta-analysis. J Trauma Stress 2013;26:299-309.

15. McFarlane AC. The longitudinal course of posttraumatic morbidity the range of outcomes and their predictors. J Nerv Ment Dis $1988 ; 176: 30-9$.

16. Jarero I, Uribe S. The EMDR protocol for recent critical incidents: Brief report of an application in a human massacre situation. EMDR Pract Res 2011;5:156-65.

17. Jarero I, Uribe S. The EMDR protocol for recent critical incidents: Follow-up report of an application in a human massacre situation. EMDR Pract Res 2012;6:50-61.

18. Van der Kolk BA, van der Hart O. (The intrusive past: The flexibility of memory and the engraving of trauma). Am Imago 1991;48:425-54.

19. Hedtke KA, Ruggiero KJ, Fitzgerald MM, et al. A longitudinal investigation of interpersonal violence in relation to mental health and substance use. J Consult Clin Psychol 2008;76:63347.

20. Andreski P, Chilcoat H, Breslau N. Post-traumatic stress disorder and somatization symptoms: a prospective study. Psychiatry Res 1998;79:131-8.

21. Vieweg WV, Julius DA, Bates J, et al. Posttraumatic stress disorder as a risk factor for obesity among male military veterans. Acta Psychiatr Scand 2007;116:483-7.

22. Scully PJ. Taking care of staff: A comprehensive model of support for paramedic and emergency medical dispatchers. Traumatology 2011;17:35-42.

23. World Health Organization. Oral health surveys: basic methods. Geneva: World Health Organization; 2013.

24. Shapiro F. Eye movement desensitization and reprocessing: Basic principles, protocols, and procedures. 2nd ed. New York, NY: Guilford Press; 2001.

25. Shapiro F. EMDR as an Integrative Psychotherapy Approach: Experts of Diverse Orientations Explore the Paradigm Prism. APA; 2002.

26. Van der Kolk BA. The assessment and treatment of complex PTSD. Treating trauma survivors with PTSD 2002;127:156.

27. Bergmann U. Speculations on the neurobiology of EMDR. Traumatology 1998;4.

28. Bergmann U. EMDR's neurobiological mechanisms of action: A survey of 20 years of searching. J EMDR Pract Res 2010;4:22-42.

29. Stickgold R. EMDR: A putative neurobiological mechanism of action. J Clin Psychol 2002;58:61-75.

30. Shapiro F. Eye movement desensitization and reprocessing: Basic principles, protocols and procedures. New York, NY: Guilford Press; 1995.

31. Wilson S, Becker LA, Tinker RH. Eye movement desensitization and reprocessing (EMDR): Treatment for psychologically traumatized individuals. J Consult Clin 1995;63:928-37.

32. Wilson S, Becker LA, Tinker RH. Fifteen-month follow-up of eye movement desensitization and reprocessing (EMDR) treatment of post-traumatic stress disorder and psychological trauma. J Consult Clin 1997;65:1047-56.

33. Van der Kolk B, Spinazzola J, Blaustein M, et al. A randomized clinical trial of EMDR, fluoxetine and pill placebo in the treatment of PTSD: Treatment effects and long-term maintenance. J Clin Psychiat 2007;68:37-46.

34. Rothbaum BO, Astin MC, Marsteller F. Prolonged exposure versus eye movement desensitization and reprocessing (EMDR) for PTSD rape victims. J Trauma Stress 2005;18:60716.

35. Bisson J, Andrew M. Psychological treatment of post-traumatic stress disorder (PTSD). Cochrane Db Syst Rev 2007;3.

36. Bradley R, Greene J, Russ E, et al. A multidimensional metaanalysis of psychotherapy for PTSD. Am J Psychiat 2005;162:214-27.

37. Davidson PR, Parker KCH. Eye movement desensitization and reprocessing (EMDR): A meta-analysis. J Consult Clin 2001;69:305-16.

38. Maxfield L, Hyer LA. The relationship between efficacy and methodology in studies investigating EMDR treatment of PTSD. J Clin Psychol 2002;58:23-41.

39. Van Etten ML, Taylor S. Comparative efficacy of treatments for post $\square$ traumatic stress disorder: A meta $\square$ analysis. Clin Psychol Psychot 1998;5:126-44.

40. Arabia E, Manca ML, Solomon RM. EMDR for survivors of life-threatening cardiac events: Results of apilot study. J EMDR Pract Res 2011;5:2-13.

41. De Roos C, Greenwald R, den Hollander-Gijsman M, et al. A randomised comparison of cognitive behavioural therapy (CBT) and eye movement desensitisation and reprocessing (EMDR) in disaster-exposed children. Eur J Psychotraumatol 2011;2:10.

42. Nijdam MJ, Gersons BP, Reitsma JB, et al. Brief eclectic psychotherapy v. eye movement desensitisation and reprocessing therapy for post-traumatic stress disorder: randomised controlled trial. Br J Psychiatry 2012;200:224-31.

43. Marcus S, Marquis P, Sakai C. Three-and 6-Month Follow-Up of EMDR Treatment of PTSD in an HMO Setting. Int J Stress Manage 2004;11:195.

44. Mitchell JT, Everly GS. The basic critical incident stress management course: Basic group crisis intervention. 3rd ed. Ellicott City, MD: International Critical Incident Stress Foundation; 2001.

45. Quinn G. Emergency Response Protocol (ERP) presentation. Paris: EMDR Europe Association Conference; 2007.

46. Quinn G. The emergency response protocol (ERP). In: Luber $\mathrm{M}$, eds. Eye movement desensitization and reprocessing (EMDR) scripted protocols: Basics and special situations. New York, NY: Springer Publishing; 2009, pp 271-276.

47. Shapiro E, Laub B. Early EMDR Intervention (EEI): A Summary, a Theoretical Model, and the Recent Traumatic EpisodeProtocol (R-TEP). J EMDR Pract Res 2008;2:79-96.

48. Tarquinio C, Brennstuhl MJ, Reichenbach S, et al. Early treatment of rape victims: Presentation of anemergency EMDR 
protocol. Sexologies 2012;21:113-21.

49. Jarero I, Artigas L, Luber M. The EMDR protocol for recent critical incidents: Application in a disaster mental health continuum of care context. J EMDR Pract Res 2011;5:82-94.

50. Silver SM, Rogers S. Light in the heart of darkness: EMDR and the treatment of war and terrorism survivors. New York, NY: Norton; 2002.

51. Chemtob CM, Nakashima J, Carlson JG. Brief-treatment for elementary school children with disaster-related PTSD: A field study. J Clin Psychol 2002;58:99-112.

52. American Psychiatric Association. Practice guideline for the treatment of patients with acute stress disorder and posttraumatic stress disorder. Arlington, VA: American Psychiatric Association; 2013.

53. Chambless DL, Baker MJ, Baucom DH, et al. Update on empirically validated therapies, II. Clin Psychol 1998;51:3-16.

54. Foa EB, Keane TM, Friedman MJ, Cohen JA. Effective treatments for PTSD: Practice guidelines from the international society for traumatic stress studies. New York, NY: Guildford Press; 2008.

55. Bleich A, Kotler M, Kutz I, Shalev A. A position paper of the (Israeli) National Council for Mental Health: Guidelines for the assessment and professional intervention with terror victims in the hospital and in the community. Jerusalem, Il: National Council for Mental Health; 2002.

56. Fernandez I, Gallinari E, Lorenzetti A. A school- based EMDR intervention for children who witnessed the Pirelli building airplane crash in Milan, Italy. J Brief Ther 2004;2:129-36.

57. Jarero I, Artigas L, Mauer M, et al. Children's post-traumatic stress after natural disasters: Integrative treatment protocol. Poster session presented at the annual meeting of the International Society for Traumatic Stress Studies. Miami, FL: International Society for Traumatic Stress Studies; 1999.

58. Fernandez I. EMDR as treatment of post-traumatic reactions: A field study on child victims of an earthquake. Educ Child Psychol 2007;24:65-72.

59. Shapiro E, Laub B. The recent traumatic episode protocol (RTEP): An integrative protocol for early EMDR intervention (EEI). In: Luber M, eds. Implementing EMDR early mental health interventions for man-made and natural disasters: Models, scripted protocols, and summary sheets. New York, NY: Springer Publishing; 2014, pp 193-215.

60. Shapiro E. EMDR and early psychological intervention following trauma. Eur Rev Appl Psychol 2012;62:241-51.

61. Weiss DS, Marmar CR. The Impact of Event Scale-Revised. In Wilson JP, Keane MT, Eds. Assessing psychological trauma and PTSD: A handbook for practitioners. New York: Guilford Press; 1997, pp 399-411.

62. Rodenburg R, Benjamin A, de Roos C, et al. Efficacy of EMDR in children: a meta-analysis. ClinPsychol Rev 2009;29:599-606.

63. Gillies D, Taylor F, Gray C, et al. Psychological Therapies for the Treatment of Post-Traumatic Stress Disorder in Children and Adolescents (Review). Evid-Based Child Health 2013;8:1004-16.

64. The ISTSS PTSD Prevention and treatment guidelines. Methodology and Recommendations and Position papers on Complex PTSD (Oct. 2018).

65. Shapiro E, Laub, B. Early EMDR intervention following a community critical incident: A randomized clinical trial. J EMDR Pract Res 2015;9:17-27.

66. Saltini A, Rebecchi D, Callerame C, et al. Early Eye Movement Desensitisation and Reprocessing (EMDR) intervention in a disaster mental health care context. Psychol Health Med 2017 [Epub ahead of print].

67. Shapiro F, Laliotis D. EMDR and the Adaptive Information Processing Model: Integrative Treatment and Case Conceptualization. Clin Soc Work J 2011;39:191. 